\title{
Kernos
}

Revue internationale et pluridisciplinaire de religion grecque antique

32 | 2019

Varia

\section{Revue des revues}

\section{Hélène Collard et Zoé Pitz}

Édition électronique

DOI : 10.4000/kernos.3336

ISSN : 2034-7871

\section{Éditeur}

Centre international d'étude de la religion grecque antique

\section{Édition imprimée}

Date de publication : 1 décembre 2019

Pagination : 379-392

ISBN : 978-2-87562-229-7

ISSN : 0776-3824

\section{Référence électronique}

Hélène Collard et Zoé Pitz, «Revue des revues », Kernos [En ligne], 32 | 2019, mis en ligne le 10 octobre 2019, consulté le 01 février 2022. URL : http://journals.openedition.org/kernos/3336 ; DOI : https:// doi.org/10.4000/kernos.3336

Ce document a été généré automatiquement le 1 février 2022.

Kernos 


\title{
Revue des revues
}

\author{
Hélène Collard et Zoé Pitz
}

1 ABE Takuji, « Proskynēsis: From a Persian Court Protocol to a Greek Religious Practice »,
Tekmeria
(2017-2018),
p. 1-45
[tente

élucider la véritable nature du protocole perse appelé par les Grecs proskynēsis, et de d' montrer pourquoi ces derniers étaient si réticents à accomplir cet acte devant le roi - un homme et non un dieu].

2 ACÉl Zsolt, « La figura di Proteo, il testo proteiforme e la struttura delle Metamorfosi (Ov. Met. 8.730-737)», Prometheus 44 (2018), p. 176-196.

3 ADORJÁNi Zsolt, « Der Gott und der König Bemerkungen zu Pindar, Kallimachos, Cicero und den Neupythagoreern Ekphantos und Diotogenes ", Hermes 146-4 (2018), p. 392414.

4 AGER Britta, « Necromancy, Divine Encounters, and Erotic Magic in Cupid and Psyche », AJPh 140-2 (2019), p. 317-343.

5 ALTRIPP Ina, «Meter und Trophos. Neue Beobachtungen an zwei großformatigen Sitzfiguren aus Pergamon »,AA (2017-2), p. 49-75.

6 AMSLER Monika, « How Could Religion Become a Category? Accounting for Classical and Fuzzy Logic in the Conceptualization of Religion », Asdiwal 12 (2017), p. 37-51 [analyse les modalités qui permettent de transformer la « religion » en une catégorie et le rôle joué par la catégorisation dans la recherche et l'épistémologie modernes].

7 ANGIÒ Francesca, «Qualche osservazione su un epigramma da Keramos con Ermes EПHK OO $\Sigma$ », PP 72-1 (2017), p. 127-140 [cette épigramme est l'occasion de discuter de l'usage

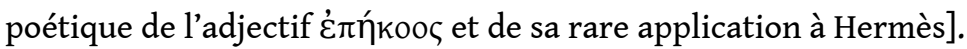

8 AZZERLINI Antonio, "Atena a Delo in Elio Aristide ", Prometheus 45 (2019), p. 192-204 [analyse de l'importance d'Athéna et de ses différentes fonctions dans l'œuvre d'Aristide, notamment dans les discours 37 (Hymne à Athéna) et 44 (Hymne à la mer Égée), avec un focus particulier sur les liens qu'entretenait la déesse avec l'île de Délos].

9 BADOUD Nathan, « L’Apollon de Piombino », JS (2018-2), p. 185-288. 

p. 279-306 [la prise en compte du contexte historique et topographique conduirait à penser que la Victoire de Samothrace a été élevée en 197 av. J.-C., au terme de la deuxième guerre de Macédoine, pour commémorer la défaite de Philippe $V$ à la bataille de Chios, quatre ans plus tôt]. [discute deux passages littéraires (Apoll. Rhod. Arg. 4.1413 et Anth. Gr. 16.91.3) renvoyant à la version selon laquelle les $\mu \tilde{\eta} \lambda \alpha$ d'or gardées par les Hespérides ne seraient pas des pommes, mais des brebis].

BARBARA Sébastien, «Héraclès, les satyres et le serpent : à propos des Epitainarioi de Sophocle ", Pallas 108 (2018), p. 169-183 [fait le point sur les Epitainarioi, un drame satyrique perdu de Sophocle, en examinant le cadre chronologique et historique ainsi que ses thématiques et son éventuel synopsis et en utilisant des échos décelables chez Aristophane]. BARCAT Dominique, «L'usage funéraire des amulettes en Grèce et en Égypte aux époques géométrique et archaïque : étude comparative ", Archimède [en ligne] 6 (2019). BARFOED Signe, « The Use of Miniature Pottery in Archaic-Hellenistic Greek Sanctuaries. Considerations on Terminology and Ritual Practice », Opuscula 11 (2018), p. 111-126 [propose une terminologie pour la poterie miniature, un groupe de matériel archéologique relativement négligé et souvent considéré comme non fonctionnel et sans importance].

BEDNAREK Bartłomiej, «The (Alleged) Sacrifice and Procession at Rural Dionysia in Aristophanes' Acharnians », Hermes 147-2 (2019), p.143-152 [revient sur la lecture traditionnelle de la scène des Dionysies rurales dans les Acharniens d'Aristophane]. BEDNAREK Bartłomiej, « Pelting Pentheus: On the Use of Oulai in Sacrificial Ritual », CPh 114-1 (2019), p. 144-153.

BERNABÉ Alberto, «La cosmogonía de las Metamorfosis de Ovidio y las Rapsodias órficas », Emerita 86-2 (2018), p. 207-232. BIARD Guillaume, KALLIONTZIS Yannis, CHARAMI Alexandra, «La base des Muses au sanctuaire de l'Hélicon ", BCH 141-2 (2017), p. 697-752 [première publication complète d'un monument consacré par les Thespiens aux Muses dans leur sanctuaire au pied de l'Hélicon, dont les éléments constitutifs sont pourtant connus depuis le XIXe siècle]. BIÈVRE-PERRIN Fabien, FINCKER Myriam, «Le tombeau de la contrada Faraone à Locres : une documentation inédite pour l'étude desnaiskoide Grande Grèce »,MEFRA [en ligne] 130-1 (2018) [l'étude approfondie d'un fragment architectural ayant appartenu à un naiskosfunéraire duIv siècle av. J.-C. permet aujourd'hui de proposer une restitution fiable, rarement envisageable au vu du reste de la documentation archéologique, et d'aborder ce type d'édifices d'un point de vue historique et sociologique].

BONNARD Jean-Baptiste, «L'exposition des nouveau-nés handicapés dans le monde grec, entre réalités et mythes : un point sur la question ", Pallas 106 (2018), p. 229-240 [fait le point sur l'historiographie de l'exposition des enfants nouveau-nés handicapés, tant dans les realia que dans les mythes; dans les nombreux mythes qui touchent à l'exposition d'enfants nouveau-nés, seuls ceux d'ÆEdipe et d'Héphaïstos ou ceux 
concernant des jumeaux semblent établir un rapport entre l'exposition d'un ou deux garçons avec un éventuel handicap].

BOSNAKIS Dimitris, HALloF Klaus, "Alte und neue Inschriften aus Kos V ", Chiron 48 (2018), p. 143-158 [édition d'une stèle opistographe mise au jour en 2009 entre la ville de Cos et l'Asclépieion; le recto contient la fin d'un calendrier sacrificiel relatif au culte de Rhéa (milieu du III siècle av. J.-C.) ; sur le verso sont gravés des règlements relatifs à des sacrifices en l'honneur d'une divinité inconnue (début du III siècle av. J.-C.); édition d'un nouveau fragment non jointif de IG XII 4, 289 avec des règlements pour l'Asclépieion]. воuillot Kevin, « Agir d'après les dieux. Lexique et représentation de l'action rituelle

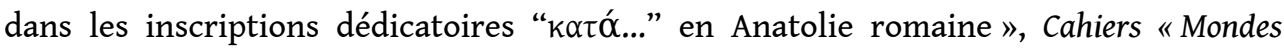
anciens» [en ligne] 12 (2019) [étude contextuelle de la documentation épigraphique anatolienne relative aux dédicaces effectuées «d'après un ordre/commandement/ rêve » attribué à un dieu].

BRAVo Benedetto, «Encore sur le katadesmosdu banquier Pasiôn : un postscriptum », BCH 141-2 (2017), p. 659-667 [nouvelle lecture de la tablette de plomb qui a fait l'objet d'un article du BCH139-140 (2015-2016), p.211-236, proposée sur la base d'une vieille photographie retrouvée récemment].

BREKKE Stewart E., "Elements of Ouranic and Chthonic Worship in Minoan Crete, Especially on the Hagia Triada Sarcophagus ", AncW 47-2 (2017), p. 115-122.

BREMMER Jan N., " Method and Madness in the Study of Greek Shamanism: The Case of Peter Kingsley", Asdiwal 13 (2018), p. 93-109 [il semble qu'il n'existait pas de chamanisme grec ni d'influence d'Asie centrale].

BRILLANTE Carlo, «Il sogno di Clitennestra nell'Oresteadi Stesicoro e nelle Coeforedi Eschilo ", QUCC 119-2 (2018), p.11-39 et ID., "Il sogno di Clitennestra nell'Elettradi Sofocle », QUCC 122-2 (2019), p. 33-50 [deux articles qui examinent les différentes versions du songe de Clytemnestre (respectivement chez Stésichore, Eschyle et Sophocle)].

BRISSON Pierre-Luc, "Antiochos IV et les festivités de Daphnè : aspects de la politique séleucide sous l'unipolarité romaine», REG 131-2 (2018), p. 415-449 [analyse la politique extérieure d'Antiochos IV Épiphane durant cette période; réaffirme le jugement posé par Polybe, qui considérait que les fêtes panhelléniques organisées par É piphane à Daphnè en 166 av. J.-C. visaient à surpasser les festivités organisées l'année précédente à Amphipolis par Paul-Émile]. : constructions sensorielles et expériences du divin dans les épopées homériques », Mythos 11 (2017), p. 83-93. BROUILLET Manon, CARASTRO Cléo, "Parures divines. Puissances et constructions homériques de l'objet ", Mètis n.s. 16 (2018), p. 85-106 [s'interroge sur la manière dont le texte homérique construit des artefacts relevant de la sphère divine, à partir des exemples de l'égide et du ruban d'Aphrodite dans l'Iliade]. CAMPBell Celia, « (Poetic) License to Kill: Apollo, the Python, and Nicander's Theriaca in Ovid, Metamorphoses $1 », G \& R$ 65-2 (2018), p. 155-174. CASAlI Sergio, « Notizie da Nestore in Ovidio, Heroides I 25-28 », Aevum Antiquum n.s. 17 (2017), p. 175-198. 
CAYLA JeanBaptiste, «Antoine, Cléopâtre, et les technites dionysiaques à Chypre ", BCH 141-1 (2017), p. 313-336 [propose une nouvelle datation pour trois dédicaces à Aphrodite Paphienne mentionnant les technites dionysiaques de Chypre; ces documents jettent un nouvel éclairage sur la période dite de la « restauration » lagide à Chypre].

CHALAZONITIS Ioannis, KOUKOULI-CHRYSANTHAKI Chaido, MALAMIDOU Dimitria, « ENTOE A M $\Omega$ MHTON: An Argive-type Shield from the Sanctuary of Oisyme ", ABSA 113 (2018), p. 199-219 [le bouclier découvert en 1987 sur l'acropole d'Oisyme (Thrace) est à présent le seul de ce type à avoir été trouvé dans un sanctuaire et le seul exemple jusqu' connu en Égée nord-orientale ; il s'agit probablement d'un don personnel, offert soit par un ancien hoplite soit en remerciement suite à une victoire militaire].

CHARAlambidou Xenia, «Iron Age Mortuary Practices and Material Culture at the Inland Cemetery of Tsikalario on Naxos: Differentiation and Connectivity », ABSA 113 (2018), p. 143-198.

CHLUP Radek, « On the Nature of the Gods: Methodological Suggestions for the Study of Greek Divinities », HR 58-2 (2018), p. 101-127 [ambitionne de proposer une nouvelle méthode pour la compréhension des dieux grecs qui s'appuie sur l'approche de JeanPierre Vernant, mais ajoute une nouvelle dimension à celle-ci].

CHRISTESEN Paul, "Typology and Topography of the Spartan Burials from the Protogeometric to the Hellenistic Period: Rethinking Spartan Exceptionalism and the Ostensible Cessation of Adult Intramural Burials in the Greek World », ABSA 113 (2018), p. 307-363 [alors que notre connaissance des pratiques funéraires à Sparte a longtemps été fondée sur les sources textuelles uniquement, cet article offre la première analyse synthétique de la typologie et de la topographie des tombes spartiates qui s'appuie sur les témoignages archéologiques].

CHRONOPOUlou Eleni, «Una inscripción mágica de Panticapeo única y los enemigos desconocidos ", Emerita 87-1 (2019), p. 73-82 [analyse d'une inscription magique de la nécropole antique de Panticapeo qui contient dix-sept fois le terme

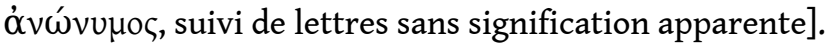

costanza S., «La tipologia divinatoria di P. Gen. inv. 161 inerente alla ieroscopia », ZPE 207 (2018), p. 5-13.

COUGHLIN Rebecca, «Uniting with Divine Wisdom: Theurgic Prayer and Religious Practice in Dionysius and Marsilio Ficino », Dionysius 36 (2018), p. 142-155.

coulié Anne et al., "Le tombeau A de Camiros: les vases archaïques et leur contenu. L'apport de l'étude chronologique et des analyses chimiques des résidus »,BCH 141-2 (2017), p. 553-621 [revient sur un contexte funéraire découvert au XIX ${ }^{e}$ siècle à Camiros par l'archéologue Auguste Salzmann et récemment reconstitué au musée du Louvre].

4 CURTIS Lauren, «Ovid's Io and the Aetiology of Lament », Phoenix 71-3/4 (2017), p. 301320 [l'épisode d'Io dans les Métamorphoses d'Ovide contient une étiologie de la pratique humaine de la lamentation].

DAVIES Malcolm, «Euripides Helen 375-85 and Sophocles Electra 150-2 as "Mythological Hyperboles" ",SCI 37 (2018), p. 1-5 [l'ancienne interprétation de ce passage comme constituant une « hyperbole mythologique » est étayée par des éléments stylistiques, et 
elle exclut en fait une lecture plus récente de la stanza qui détourne de l'effet d'hyperbole mythologique].

DE BOER Katherine R., « Pindar's Peaceful Rapes », Helios 44-1 (2017), p. 1-27 [si la piété de Pindare est bien connue, un des aspects par lesquels celle-ci se manifeste l'est moins : la façon qu'a le poète de décrire les épisodes de viols divins, qu'il remodèle en relations sexuelles agréables et réciproques pour en faire finalement des histoires d'affection et de mariage]. DEL PILAR FERNÁNDEZ DEAGUSTINI María, «La tragedia de la inmunidad. Asyliay hiketeiaen Suplicantesde Esquilo", QUCC 122-2 (2019), p. 11-33 [s'interroge sur ce qu'implique la demande de protection d'un étranger et sur la façon dont elle peut être accomplie, tant $\mathrm{du}$ point de vue du suppliant que de celui du supplié, selon les critères établis par Naiden (Ancient Supplication, 2006)].

DEMAKOPOULOU K., AULSEBROOK S., «GOLD AND SILVER VESSELS AND OTHER PRECIOUS FINDS FROM THE THOLOS TOMB AT KOKLA IN THE ARGOLID", ABSA 113 (2018), P. 119-142 [publication du mobilier funéraire de cette importante et fascinante tombe de l'Helladique récent IIB-IIIA1]. DRELIOSSI-HERAKLEIDOU Anastasia, HALLOF Klaus, «Eine neue Grenzziehungsurkunde aus Lepsia ", Chiron 48 (2018), p. 159-170 [édition d'une stèle opistographe mise au jour en 2000 à Lepsia ; sur l'avant est gravé un décret du peuple de Milet, daté de la $2^{\mathrm{e}}$ moitié du II siècle, tandis que le verso contient un acte, qualifié de $\pi$ ??????????].

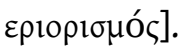
ECKERMAN Chris, "The Dioscuri and the АГ $\Omega$ N at Pindar's Olympian 3.36 ", RhM 161-1 (2018), p. 109-111 [suggère que, dans le passage en question, Héraclès ne confie pas aux Dioscures la supervision des jeux olympiques en général, mais plus précisément celle de la course de chars]. ECKERMAN Chris, "Lucretius on the Divine. DRN3.17-30, 5.1161-93, and 6.68-79", Mnemosyne 72-2 (2019), p. 284-299. EIDINOW Esther, " "The Horror of the Terrifying and the Hilarity of the Grotesque": Daimonic Spaces - and Emotions - in Ancient Greek Literature », Arethusa 51-3 (2018), p. 209-235.

51 EIDINOw Esther, «The (Ancient Greek) Subject Supposed to Believe », Numen 66-1 (2019), p. 56-88 [discute des difficultés auxquelles font face les chercheurs qui s'intéressent à la nature $\mathrm{du}$ « croire » dans la religion grecque ancienne]. EISENFELD Hanne, "Geryon the Hero, Herakles the God», JHS 138 (2018), p. 80-99 [réévalue la fonction narrative de Géryon et d'Héraclès dans la Géryonide de Stésichore à la lumière d'opinions contemporaines sur l'apothéose d'Héraclès].

EKROTH Gunnel, «Why Does Zeus Care about Burnt Thighbones from Sheep? Defining the Divine and Structuring the World through Animal Sacrifice in Ancient Greece », HR 58-3 (2019), p. 225-250 [étudie le sacrifice animal en Grèce ancienne du viII ${ }^{\mathrm{e}}$ au I ${ }^{\mathrm{er}}$ siècle av. J.-C., en se focalisant sur trois aspects en particulier: les différents types de rituels impliquant les animaux; la manière dont le sacrifice constituait un moyen de définir les dieux; la manière dont la manipulation de la viande lors du sacrifice animal structurait le monde en marquant des hiérarchies et des distinctions de statut]. ELDEM Edhem, «Et si Diane n'était pas lesbienne ? Biographie critique d'une statue(tte) d'Artémis du Musée archéologique d'Istanbul », BCH 141-1 (2017), p. 389-448 [propose une relecture des interprétations et commentaires dont fut l'objet cette célèbre statue 
d'Artémis, cataloguée comme provenant de Lesbos vers 1865, mais dont il est désormais certain qu'elle fut découverte en 1844 au lieudit des "Bains de Diane" (Halkapınar) dans les environs de Smyrne].

ERICKSON Kyle, " Another Century of Gods? A Re-evaluation of Seleucid Ruler Cult », CQ 68-1 (2018), p. 97-111 [les rois séleucides auraient été considérés comme de nature divine avant le règne d'Antiochos III déjà, bien qu'il n'y ait pas eu de culte du souverain établi et centralisé comme c'était le cas pour les Ptolémées].

FACHARD Sylvian et al., "La Nécropole du Canal à Érétrie : topographie et inscriptions ", BCH 141-1 (2017), p. 141-226 [à l'automne 2009, à Érétrie, plusieurs tombes et stèles funéraires apparurent dans le lit du ruisseau bordant le site à l'Ouest ; ces découvertes apportent un éclairage bienvenu sur les nécropoles érétriennes, encore mal connues].

FOLEY Elizabeth, STROUD Ronald S., «A Reappraisal of the Athena Promachos Accounts from the Acropolis (IG I $\left.\mathrm{I}^{3} 435\right)$ », Hesperia 88-1 (2019), p. 87-153 [nouvelle édition de IG I $\mathrm{I}^{3}$ 435 , généralement identifiée comme des comptes relatifs à la création de la grande statue en bronze d'Athéna Promachos sur l'Acropole; l'analyse des sources littéraires, épigraphiques et archéologiques en lien avec la statue d'Athéna Promachos suggère que les arguments qui permettent d'associer l'inscription à cette statue ne sont pas convaincants et qu'une autre association doit être proposée].

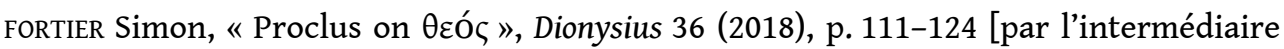
de Proclos et de son analyse du terme $\theta \varepsilon o ́ \varsigma$, s'intéresse à la façon dont les Grecs parlaient de leurs dieux, toujours dans une curieuse juxtaposition de clarté et d'ambiguïté].

FOUQUET J., KATÓ P., « Gefallen im Krieg: ein späthellenistischer Rundaltar aus Troizen », ZPE 207 (2018), p. 47-53 [publication et commentaire d'une inscription gravée sur un autel rond mis au jour à Trézène].

FrancobandiERA Daria, "Le retour des Érynies: le chœur des Euménides dans Les Mouches de Jean-Paul Sartre et La Ville parjure d'Hélène Cixous », Pallas 108 (2018), p. 7991.

61 FRIES Almut, «Pindar, Pae. 6, 121-122. Measuring Song and Sacrifice in Greek Lyric and the Rigveda», QUCC 121-1 (2019), p. 33-50 [étant donné qu'elle présente plusieurs similitudes avec des éléments du Rig-Veda, l'invocation des vers 121-122 du Sixième Péan de Pindare pourrait être une expression d'origine indo-européenne ou, au moins, gréco-aryenne].

FURLEY W., GYSEMBERGH V., « A New Edition of P.Ross.Georg. I 21 », ZPE 208 (2018), p. $94-$ 102 [présentation des résultats du réexamen - possible grâce à l'autopsie et aux photographies - du papyrus P.Ross.Georg. I 21, publié précédemment dans Reading the Liver. Papyrological Texts on Ancient Greek Extispicy]. GaGLiANo Elena, "Heracles, Theseus and Apollo anadoumenos ten komen. Three 'Forgotten< Statues from the Athenian Agora », MDAI(A) 133 (2018), p. 95-125 [propose une comparaison entre ces trois statues, qui n'ont jamais fait l'objet d'une analyse, et un relief athénien bien connu, exposé au musée Barracco à Rome]. GHEERBRANT Xavier, "Théorie poétique, vérité et représentation du divin chez Empédocle et Pindare », Pallas 108 (2018), p. 211-224. GILLMEISTER Andrzej, musiat Danuta, "Why Were the "Oriental Cults" Invented? », AAntHung 57-4 (2017), p. 503-513 [analyse le contexte historiographique de la catégorie 
des dits "cultes orientaux" ou «religions orientales", créée au XIX siècle et développée par Franz Cumont].

GIORGIANNI Franco, " Technaie prerogative divine sulla scena del teatro di Eschilo », Mythos 11 (2017), p. 131-144.

GKIKAKI Mairi, « Das Amphiglyphon der Akademie », MDAI(A) 133 (2018), p. 127-145 [sur

l'interprétation de l'amphiglyphon mis au jour dans la zone de l'Académie de Platon; la face A montre Athéna comme protectrice de la cité ; la face B est difficile à interpréter en raison de son caractère fragmentaire, mais pourrait représenter une centauromachie].

GOURMELEN Laurent, « L'apport des scholies homériques (scholies D) à la connaissance du mythe de Sisyphe : transmission d'un récit de Phérécyde d'Athènes (fragment 119)", RPh 90-2 (2016), p. 111-128.

GRAND-CLÉMENT Adeline, " “Il est interdit de...". Rituels et procédures de régulation sensorielle dans le monde grec ancien : quelques pistes de réflexion", Mythos 11 (2017), p. 49-58 [envisage les prescriptions rituelles sous l'angle des effets qu'elles avaient sur les membres de la communauté qui s'y soumettaient].

GRECO Giovanna, «Ecate a Velia », PP 72-1 (2017), p. 141-157 [à propos d'une statue en marbre d'Hécate trouvée à Vélia/Élée et du type iconographique auquel elle appartient, qui, selon Pausanias, aurait été créé par Alcamène].

GUIMIER SORBETS Anne-Marie, « Des emblemata - pinakes déliens : iconographie, technique, usage ", $B C H$ 141-1 (2017), p. 227-263 [envisage l'iconographie, la technique et l'usage de trois panneaux mosaïqués (fin $\mathrm{II}^{\mathrm{e}}$ - début $\mathrm{I}^{\mathrm{er}}$ siècle) découverts à Délos, dont deux proviennent d'un sanctuaire (le Kynthion et le Samothrakeion) et le troisième d'une maison].

HALLMANNSECKER M., " Heracles Hoplophylax, Iudaioi, and a Palm Grove. A Fresh Look at I.Smyrna 497 », EA 50 (2017), p. 109-127 [nouvelle édition et commentaire de l'inscription].

HERKLOTZ Friederike, «Vom ägyptischen Lokalgott zur griechischen Universalgottheit Sarapis als Heil- und Orakelgott in der Prolemäerzeit », AncSoc 48 (2018), p. 9-48 [étudie la manière dont Sarapis est devenu un dieu oraculaire et guérisseur durant la période ptolémaïque ; cherche à comprendre comment un dieu d'origine égyptienne a pu être si populaire chez les Grecs].

4 HILTON Ita, "The "Sown Men" and the Sons of Oedipus: Representations of Land, Earth and City in Euripides' Phoinissai»,Hermes 146-3 (2018), p. 263-276 [aborde les représentations de Thèbes dans les deux mythes centraux des Phéniciennes : celui de l'autochtonie et de la famille d'ÆEdipe].

5 HORN Fabian, « Dying is Hard to Describe: Metonymies and Metaphors of Death in the Iliad », CQ 68-2 (2018), p. 359-383.

6 HOz M.-P. (DE), «The Regulations of Dionysius in the So-Called lex sacra from Philadelphia in Lydia. Elevated Strict Moral Code or Current Civil Behavioural Norm? ", EA 50 (2017), p. 93-108 [étudie l'inscription d'un point de vue différent de celui qui a déjà été proposé par d'autres chercheurs ; l'approche de l'auteure est proche de celle de Stower, mais le contexte social dans lequel elle l'analyse et l'interprète est différent].

HULME KOZEY Emily, «Another peri technes Literature: Inquiries about One's Craft at $\begin{array}{lllll}\text { Dodona } ", ~ G \& R & 65-2 & \text { (2018), 205-217 }\end{array}$ 
étude du corpus des lamelles oraculaires de Dodone, vise à apporter un nouvel éclairage l' à notre connaissance de la techne ancienne : art, artisanat, profession]. (2019), p. 498-506 [considère deux passages poétiques - l'un de Callimaque et l'autre de Catulle - qui peuvent refléter l'affirmation de G. Nagy selon laquelle, pour les érudits hellénistiques, l'idée d'Océan comme un principe cosmologique était associée à la poésie orphique].

JAMme Christoph, «Liebe im Paramythos. Zu Rilkes Gestaltung des Orpheus-Mythos », Philotheos 18 (2018), p. 305-313.

JAUPAJ Lavdosh, «Une tombe hellénistique de Gjerbës (Albanie): un marqueur culturel? », BCH 141-1 (2017), p. 287-311 [retrouvée sur le territoire du Koinon des Bylliones, dans l'arrière-pays d'Apollonia, épitaphe de Megallis non seulement enrichit le corpus des inscriptions grecques de la l' région, mais pose aussi le problème de l'identité des occupants de la nécropole].

Jost Madeleine, "Sanctuaires d'Arcadie trente ans après : bilan des recherches ", BCH 142-1 (2018), p. 97-143 [dresse le bilan des recherches sur les sanctuaires depuis la parution de l'ouvrage Sanctuaires et cultes d'Arcadiepublié par l'auteure en 1985].

JURAJ Franek, "Invocations of the Muse in Homer and Hesiod: A Cognitive Approach », Antichthon 52 (2018), p. 1-22 [propose une analyse cognitive des invocations de la Muse dans la poésie épique grecque basée sur des avancées récentes dans le domaine des sciences cognitives en général et dans le domaine des sciences cognitives de la religion en particulier].

Kanellopoulos Chrysanthos, Petrakis Manolis, «Cella Alignment and 4th Century BC Doric Peripteral Temple Architecture in Mainland Greece ", Opuscula 11 (2018), p. 169200 [propose une catégorisation des temples doriques périptères et analyse en détail cinq temples périptères : le temple d'Asclépios à Gortyne, le temple d'Apollon Ismenios à Thèbes, le temple d'Apollon au Mont Ptoion et le temple dit "d'Hippolyte » à Troizen].

KANSTEINER Sascha, « Der Hermes Typus Richelieu », AA (2017-2), p. 77-98.

KARATAS A.M.S., " The Significance of Money for the Cults and Sanctuaries of Demeter and Kore: The Shaping of the Cults by Commercial Transactions ", Mythos 12 (2018), p. 47-88 [sur la monétarisation des cultes de Déméter].

KARETSOU Alexandra, merousis Nikos (trad. de Béatrice DEtournaY), "Sépulture "de guerrier" dans une tombe à chambre Minoen Récent IIIA2-B de Galia, Messara (Crète) ", BCH 142-1 (2018), p. 1-47 [le mobilier funéraire de la tombe de Galia, fouillée en 1975, montre que les pratiques funéraires de la phase palatiale finale se poursuivaient dans la Crète post-palatiale et prouve de façon indéniable que les élites locales voulaient, de cette manière, afficher leur statut social].

KAYACHEV Boris, «Oceanus and Orphic Allusion in Callimachus and Catullus », CPh 114-3 KIENAST Herrman J., FURTWÄNGLER Andreas E., « Zur Datierung der beiden Dipteroi im Heraion von Samos. Die Ergebnisse der Ausgrabung von 1989 », MDAI(A) 133 (2018), p. 59-94.

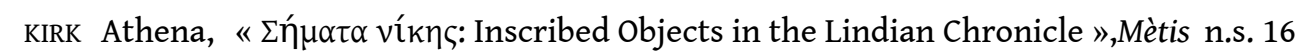
(2018), p. 107-124 [partant de l'idée que la Chronique de Lindos met l'accent sur la 
matérialité des inscriptions, cette étude examine l'interaction entre objet et texte dédicatoires dans le contexte particulier où objets et textes ont disparu].

KLINGER Sonia, "Terracotta Models of Sandaled Feet: Votives from the Sanctuary of Demeter and Kore on Acrocorinth", Hesperia 87-3 (2018), p. 429-449 [publication de quatre modèles en terre cuite représentant des pieds chaussés de sandales provenant du sanctuaire de Déméter et Korè sur l'Acrocorinthe].

KLONIZAKI Ariane, "Trouvailles monétaires à l' "édifice E" de l'Asklépieion d'Épidaure. Précieux témoignages pour l'étude d'un important lieu de culte», $B C H$ 139/140-2 (2016), p. 553-564 [l'étude des monnaies del'Asklépieion Épidaure contribue à notre compréhension de ce lieu de culte et de son évolution : d' provenant d'ateliers péloponnésiens, elles ont pour la plupart été retrouvées avec d'autres offrandes et attestent notamment l'origine variée des pèlerins]. Kosmopoulos Dimosthenis, «Kos, "santuario del porto". Analisi architettonica e relazioni tra diversi linguaggi stilistici »,ASAA 96 (2018), p. 149-173. котшіск Mirjam E., « АNOHTOI АмҮHTOI: Allegorical Interpretation in the Derveni Papyrus and Plato's Gorgias »,CPh 114-2 (2019), p. 173-196 [le parallèle entre l'allégorie des jarres qui fuient, mentionnée dans un passage du Gorgias de Platon, et le papyrus de Derveni est tout à fait significative : la méthode de l'interprétation allégorique et son objectif moral semblent être les mêmes dans les deux cas].

KRISTOFFERSEN Tore R., «The Artemis Orthia Inscriptions and Spoken Laconian in the Imperial Period: In Defence of Dialect Survival at Sparta ", Glotta 95-1 (2019), p. 169-189 [un petit nombre d'inscriptions venant du sanctuaire d'Artémis Orthia à Sparte et datant

époque impériale font usage du dialecte laconien, ce qui a souvent été considéré l' comme une expression d'

« antiquarianisme » : le inscriptions of

LARDINOIS André, « Eastern Myths for Western Lies. Allusions to Near Eastern Mythology in Homer'slliad », Mnemosyne 71-6 (2018), p. 895-919 [si l'influence du Proche Orient sur la culture grecque mycénienne et archaïque a été largement démontrée par l'archéologie, cette influence étendait-elle aussi à la littérature ? Et était-elle reconnue par les anciens Grecs eux$\mathrm{s}^{\prime}$ mêmes ?]

LATIFSES Ajda, «Le sang et l'eau. Du sacrifice à la purification dans Iphigénie en Tauride d'Euripide ", Mètis n.s. 16 (2018), p. 335-355 [met en évidence l'importance du thème rituel dans la construction dramaturgique et poétique de l'Iphigénie en Tauride et dans la signification de la pièce]. LAUGHY Michael, «Figurines in the Road: A Protoattic Votive Deposit from the Athenian Agora Reexamined », Hesperia 87-4 (2018), p. 633-679 [un réexamen du dépôt et de son contexte suggère que les offrandes mises au jour ne sont pas liées à un culte de la mort, mais sont des dédicaces à Déméter et proviennent de l'Éleusinion]. LAUGHY Michael, "The Origins of the Democratic Priestess of Athena Nike ", Historia 674 (2018), p. 418-433 [sur la prêtrise d'Athèna Nikè qui, auparavant entre les mains d'un genos, en vint à être contrôlée par l'état]. LAVILLA DE LERA Jonathan, "The Prayer to Pan of Plato's Phaedrus (279b8-c3): An Exhortation to Exercise the Philosophical Virtue »,SO 92 (2018), p. 65106 [offre une nouvelle lecture de la prière adressée à Pan par Socrate et montre, par 
une approche globale, que celle-ci exhorte en fait l'auditeur à s'engager dans le mode de vie décrit par le personnage de Socrate, à savoir celui de la philosophie].

LEIGH CLAYTON Barbara, « Heracles, Hylas, and the Homeric Hymn to Demeterin Apollonius's Argonautica ", Helios 44-2 (2017), p. 133-156 [de nombreux détails dans le récit de la disparition d'Hylas, racontée au livre I des Argonautiques, indiqueraient qu'Apollonios fait délibérément le rapprochement entre cet épisode et celui de l'enlèvement de Perséphone dans l'Hymne homérique à Déméter].

LESSER Rachel H., " The Pandareids and Pandora: Defining Penelope's Subjectivity in the Odyssey ", Helios 44-2 (2017), p. 101-132 [la prière de Pénélope à Artémis, au cours de laquelle elle relate l'histoire des Pandarides (Odyssée, livre XX), n'a reçu que peu d'attention de la part des modernes; pourtant, elle constituerait une voie non négligeable pour interpréter le personnage de Pénélope et son rôle narratif dans le poème].

101 LESSER Rachel H., « Female Ethics and Epic Rivalry: Helen in the Iliad and Penelope in the Odyssey ", AJPh 140-2 (2019), p. 189-226 [l'intertextualité entre Pénélope et Hélène, qui sont construites dans épopée homérique comme deux héroïnes antithétiques, contribuerait à la rivalité entre l'

et l'Iliade et pourrait être comprise comme une contrepartie féminine aux héroïsmes concurrents d'Ulysse et Achille].

102 LEWIS Virginia M., «Cultic Connections in Pindar's Nemean 1 »,CPh 114-3 (2019), p. 344365 [l'usage par Pindare de l'allusion mythique dans la première Néméenne souligne l'héritage cultuel partagé par les citoyens d'Aitna et présente la notion d'un corps citoyen cohésif aux publics de Sicile et d'ailleurs].

LIANGOURAS Christos, NTOUNTOUMI Konstantina, "Monnaies en bronze provenant d'un nouveau sanctuaire à Olympie », $B C H$ 139/140-2 (2016), p. 599-608 [la fouille d'urgence menée aux abords du stade d'Olympie en 2007 et 2008 a permis de dégager un édifice en poros, identifié au sanctuaire de Déméter Chamyné dont parle Pausanias (VI, 21, 13); cette fouille a aussi livré 33 monnaies de bronze et une monnaie fourrée]. Lightfoot Jessica, « Something to Do with Dionysus? Dolphins and Dithyramb in Pindar Fragment $236 \mathrm{SM} », C P h$ 114-3 (2019), p. 481-492 [sur le fragment $236 \mathrm{SM}$, qui dérive de la version pindarienne du récit mythique de la capture de Dionysos par des pirates, ensuite transformés en dauphins par le dieu].

LóPEZ-RUIZ Carolina, «Gargoris and Habis: A Tartessic Myth of Ancient Iberia and the Traces of Phoenician Euhemerism ", Phoenix 71-3/4 (2017), p. 265-287. MACEDO José Marcos, «Hermes a-re-ja (PY Tn 316): A New Interpretation », Kadmos 55 (2016), p. 67-82 [l'auteur propose d'interpréter la séquence a-re-ja, présente dans la tablette de Pylos Tn 316, comme un nom issu de la racine ${ }^{*} h_{2}$ leu - - éloigner »apposé au nom Hermès]. MACK William, « Vox populi, vox deorum? Athenian Document Reliefs and the Theologies of Public Inscription », ABSA 113 (2018), p. 365-398.

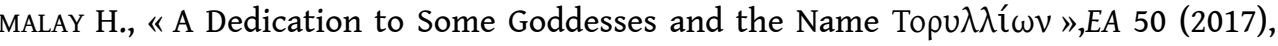
p. 25-26 [note sur une dédicace copiée dans la collection du Dr. Ü. Evran en 2000].

109 MALTOMini F., «Note al testo di alcuni papiri magici viennesi », ZPE 207 (2018), p. 115121. 
. Il contributo del paesaggio sacro alla conoscenza di unethnos epirota », ASAA 95 (2017), p. 205-225 [réévalue notre connaissance des antiquités cultuelles thesprotiennes en comparant différents types de sources, des sources littéraires et numismatiques aux témoignages archéologiques].

111 MARGARITI Katia, «Painting Early Death. Deceased Maidens on Funerary Vases in the National Archaeological Museum of Athens ", Opuscula 11 (2018), p. 127-150 [étudie l'iconographie des jeunes filles décédées peintes sur une loutrophore funéraire à figures rouges et six lécythes à fond blanc conservés au Musée national archéologique d'Athènes, tous datés du $\mathrm{v}^{\mathrm{e}}$ siècle].

112 MARTENS Brian, "The Statuary of Asklepios from the Athenian Agora ", Hesperia 87-3 (2018), p. 545-610 [identification et publication de la statuaire en marbre d'Asklépios mise au jour lors des fouilles de l'agora d'Athènes].

113 MASSA-PAIRAULT Françoise-Hélène, « Mythes et styles de l'hellénisme dans l'art funéraire de Chiusi ", RA 66-2 (2018), p. 349-388 [les urnes funéraires et sarcophages Étrurie offrent une ample matière pour aborder la question de la réception du mythe d' grec dans une société particulière et celle de l'impact des modèles hellénistiques sur une production artisanale].

114 MASSARI Pietro, «Dal Laurio a Drepane. L'Attica nelle Argonautiche di Apollonio », Aevum Antiquum n.s. 17 (2017), p. 275-322.

115 mathioudaki Iro, «The Pottery Deposit from the Houses of the Fallen Blocks and the Sacrificed Oxen at the South-Eastern Corner of the Palace of Knossos ", ABSA 113 (2018), p. 19-73 [nouvelle analyse de l'assemblage céramique déposé dans l'espace occupé par deux maisons au sein du Palais de Cnossos, dépôt qui a été crucial à Arthur Evans dans l'élaboration de sa théorie du "grand tremblement de terre" qui aurait détruit le palais].

116 MAURO Chiara Maria, "Religious Survivals in the Erechteion Area: A Diachronic Approach », DHA 45-1 (2019), p. 65-88 [vise à réexaminer le profil diachronique de l'É rechteion pour mieux comprendre la succession de ses phases et cherche des éléments de continuité à travers le temps, avec un regard particulier sur la nature du culte accompli dans la zone de l'Érechteion].

117 MAZUREK Lindsey A., "The Middle Platonic Isis: Text and Image in the Sanctuary of the Egyptian Gods at Herodes Atticus' Marathon Villa ", AJA 122-4 (2018), p. 611-644 [réexamine le programme architectural du Sanctuaire des Dieux égyptiens à Marathon en s'appuyant, d'une part, sur le public visé par ce sanctuaire - probablement des élèves et membres de l'école athénienne du moyen-platonisme -, d'autre part, sur un certain nombre de textes "isiaques", notamment le Isis et Osiris de Plutarque].

118 MCAULEY Alex, «The Tradition and Ideology of Naming Seleukid Queens », Historia 67-4 (2018), p. 472-494 [examine les méchanismes qui se trouvent derrière la répétition des noms royaux féminins; le nom Laodikè est étudié et est notamment examiné en lien avec la mythologie séleucide et le culte royal].

119 MCPHEE Brian D., « Mythological Innovations in Corinna's Asopides Poem (fr. 654.ii-iv $P M G) »$, GRBS 58-2 (2018), p. 198-222.

120 MEERT Alexander, «The Atheist Writings of Diagoras of Melos. New Approaches to the Authenticity of "Phrygioi Logoi" and "Apopyrgizontes Logoi" ", AC 87 (2018), p. 1-12 [cherche à savoir à quel degré deux titres de la production littéraire de Diagoras de 
Mélos - souvent considéré comme l'athée par excellence dans l'Antiquité classique correspondent au cadre temporel dans lequel l'auteur a vécu ou apparaissent comme provenant de traditions littéraires plus tardives]. divin », Pallas 106 (2018), p. 85-103. MEISTER Felix J., "Hieron and Zeus in Pindar ", CPh 114-3 (2019), p. 366-382 [vise à démontrer que les odes syracusaines de Pindare montrent en fait, en lien avec ce qui paraît être une interprétation d'Aristophane, une tendance générale à comparer Hieron à Zeus].

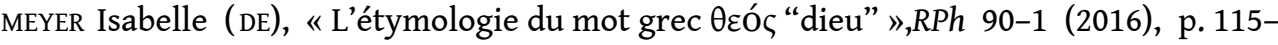
138 [discute les théories précédemment avancées sur étymologie du mot grec $\theta \varepsilon o ́ \varsigma$ et propose une analyse morphologique approfondie du l' terme]. MILES Graeme, "Mythic Paradigms and the Platonic Life: Becoming a Bacchus in Damascius'Philosophical History", JHS 138 (2018), p. 55-66. MILES HILTON Collin, «Epicurean Myth Rationalization in Plutarch's De Latenter Vivendo and Lucretius' Catalogue of Underworld Torments»,GRBS 59-1 (2019), p. 134-157 [en affirmant que le destin de l'épicurien impie n'est pas de la torture mais de l'obscurité, Plutarque utilise en l'inversant un argument trouvé chez Lucrèce]. MÖLLER Melanie, «Videri forma potest hominis » (Ovid met. I 404 ss.). Man's Creation in the Shadow of Prometheus ", Maia 70-3 (2018), p. 530-543.

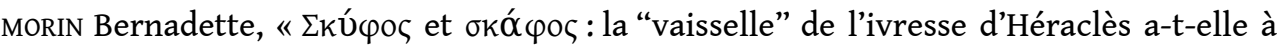
voir avec le "vaisseau" qui transporte Alceste sur l'Achéron? ", RPh 89-2 (2015), p. 3351.

MUGELLI Gloria, « "La flamme dévoratrice d'offrandes" : feu et agentivité rituelle dans la tragédie grecque ",Cahiers "Mondes anciens" [en ligne] 12 (2019) [en observant les croisements entre l'expérience du rite et l'expérience de la tragédie, cet article analyse les différentes configurations dramatiques de l'image du feu qui brûle sur l'autel]. NENCI Nicola, « The Votive of Aiglatas, Spartan Runner. Old Evidence, New Knowledge », ABSA 113 (2018), p. 251-278 [propose une nouvelle lecture et une nouvelle interprétation d'une stèle trouvée à Sparte, datant de la fin de la période archaïque et portant une dédicace à [Apollon] Karneios par Aiglatas en remerciement de ses victoires athlétiques]. NIJS Wim, " "Then Truly the Life of the Gods Will Pass to Men": Contemplating Diogenes of Oenoanda's Golden Age ", GRBS 59-2 (2019), p. 242-261 [la description d'un futur âge d'or de la tranquillité épicurienne et la doctrine de l'insouciance à propos du futur ne sont pas contradictoires si cet âge d'or est envisagé comme plaisant à imaginer plutôt que promis pour le futur].

131 NÜNLIST René, «A New Fragment of Palaephatus' Trojan History? », RhM 161-3/4 (2018), p. 433-435 [sur un passage d'un commentaire d'Eustathe citant un fragment de Palaephatus et traité par G. Hawes dans Rationalizing Myth in Antiquity]. PALMISCIANO Riccardo, « Eros in azione. Considerazioni pragmatiche sulla poesia erotica simposiale », QUCC 120-3 (2018),p. 153-176. PAPANTONIOU Giorgos, KYRIAKOU Niki, « Sacred Landscapes and the Territoriality of Iron Age Cypriot Polities: The Applicability of GIS », AJA 122-4 (2018), p. 541-577 [par le 
recours au SIG (système d'information géographique), cet article apporte une contribution au débat en cours sur la relation entre les sanctuaires et la territorialité des institutions chypriotes à l'âge du fer].

PARKER Robert, "Greek Religion 1828-2017 : the Contribution of Epigraphy », Chiron 48 (2018), p.1-15 [montre l'impact que l'afflux des témoignages épigraphiques a eu sur l'étude de la religion grecque depuis la publication du premier volume duCIG par Boeckh].

PATTONI Maria Pia, «Due esempi di ricezione omerica tra Otto e Novecento: Giovanni Pascoli e Gustav Klimt », Aevum Antiquum n.s. 16 (2016), p. 265-287.

PAVLIDES Nicolette, «The Sanctuaries of Apollo Maleatas and Apollo Tyritas in Laconia: Religion in Spartan-perioikic Relations ", ABSA 113 (2018), p. 279-305 [s'intéresse à la façon dont la religion a contribué à l'interconnectivité d'une vaste région géographique - la Laconie - qui était sous le contrôle spartiate pendant la majeure partie des périodes archaïque et classique].

PETZL G., « Im Artemis-Tempel zu Sardeis: Bitte um Schatten von Säulen? », ZPE 208 (2018), p. 138-143.

PFAFF Christopher A., «Late Antique Symbols and Numerals on Altars in the Asklepieion at Epidauros ", Hesperia 87-2 (2018), p. 387-428 [recense ce qui est connu à propos des symboles et chiffres présents sur de nombreux autels de l'Asklépieion d'Épidaure, et explore la façon dont ils pourraient avoir été utilisés. Les marques sur les autels de l'Asklépieion d'Épidaure pourraient répondre à des exigences liturgiques spécifiques à ce sanctuaire, qui demandait l'entretien régulier d'un nombre d'autels inhabituellement important].

PHILBRICK Rachel, «Coronis and the Metamorphosis of Apollo: Ovidian Re-Formations of Pindar's Third Pythian ", AJPh 139-3 (2018), p. 451-482 [s'intéresse à l'influence de Pindare sur Ovide, et notamment sur le récit que fait le poète du mythe de Coronis et de son histoire d'amour avec Apollon (Mét. 2.542-632)]. PICOT Jean-Claude, BERG William, «Apollo, Eros, and Epic Allusions in Empedocles, frr. 134 and 29 DK »,AJPh 139-3 (2018), p. 365-396. PILz Oliver, "Zwischen privat und öffentlich. Bemerkungen zum Asklepieion am Südabhang der Athener Akropolis und zum sogenannten Ärzterelief », MDAI(A) 133 (2018), p. 147-171 [considère deux exemples connexes afin d'examiner comment les sphères privée et publique s'interpénètrent dans le contexte de la pratique cultuelle grecque de la période classique].

142 POMADÈRE

Maia, «Classes d'âge et rites de passage : des catégories problématiques pour le monde égéen à l'Âge du Bronze ", $B C H$ 141-1 (2017), p.1-39 [bien que les catégories de «classes âge » et de "rites de passage » aient été largement mise en doute pour la Grèce des $d^{\prime}$ cités, elles continuent d' exclusivement fondées sur les données archéologiques]. POSAMENTIR Richard, «Die neue Hera: Ein Tempel für Livia auf Samos», MDAI(A) 133 (2018), p. 239-292 [à propos d'un petit naiskos d'ordre composite érigé dans l'Héraion de Samos au début de la période impériale à côté de deux autres bâtiments romains entre le temple archaïque et le grand autel]. 
144 PSychoyos Olga, «Monnaies provenant de tombes de la nécropole Sud de l'antique Argos ", $\quad$ BCH 139/140-2 (2016), $\quad$ p. 641-668 [l'étude des types monétaires retrouvés dans la nécropole révèle qu'ils ont été choisis, conformément croyances de époque, en raison de leur relation avec la vie après la mort : les monnaies constituent l' une forme de contrat passé entre le mort et les dieux chthoniens]. RAUHALA Marika « Arresting Alternatives. Religious Prejudice and Bacchantic Worship in Greek Literature », Numen 66-1 (2019), p. 1-55. REBER Karl et al., "Les activités de l'école suisse d'archéologie en Grèce en 2017. Le Gymnase d'Érétrie et l'Artémision d'Amarynthos ", AK 61 (2018), p. 123-137. REUSSER Christoph, «Ein attischer Falaieff-Krater aus einem etruskischen Wohnhaus. Ein Neufund aus den Zürcher Ausgrabungen in Spina ", AK 61 (2018), p. 16-31 [sur un cratère à figures rouges de la fin de la période classique mis au jour dans la cité étrusque de Spina ; des critères formels et stylistiques permettent d'attribuer le cratère au groupe dit « Helbig » et de le dater de c. 370-360 av. J.-C.]. RICL M., «Cults of Phrygia Epiktetos in the Roman Imperial Period», EA 50 (2017), p. 133-148 [analyse les cultes attestés dans la région phrygienne appelée «Phrygie Epiktetos », dont la cité principale était Aizanoi].

149 RIGSBY K.J., « Asclepius in Illyria », ZPE 207 (2018), p. 55-56 [à propos du texte inscrit sur une lamelle mise au jour à Apollonia en Illyrie, publié par P. Cabanes et repris dans la base de données Collection of Greek Ritual Norms (CGRN 40)]. ROMERo MARISCAL Lucía P., «Sappho F 44 Voigt and Euripides'Troades », Mnemosyne 71-6 (2018), p. 920-937 [propose une nouvelle interprétation du premier stasimon des Troyennes d'Euripide, fondée sur un potentiel lien avec un poème de Sappho].

151 ROSE Thomas C., « Demetrius Poliorcetes, Kairos, and the Sacred and Civil Calendars of Athens ", Historia 67-3 (2018), p. 258-287 [démontre que Démétrios Poliorcète a programmé à plusieurs reprises d'arriver à Athènes durant d'importantes fêtes religieuses ; la célébration de ces cas de kairos a inscrit Démétrios dans les calendriers de la cité et a favorisé son programme d'auto-établissement divin]. RosKAm Geert, "Philo of Alexandria on the Twelve Olympian Gods », CW 112-3 (2019), p. 169-192 [s'intéresse à un aspect souvent négligé de la pensée de Philon d'Alexandrie, à savoir son attitude vis-à-vis des dieux du panthéon grec].

153 RÜPKEJörg, « Una prospettiva individualizzata sulla religione antica »,Mythos 11 (2017), p. $145-155$.

154 SABETAI Victoria, AVRonidaki Christina, "The Six's Technique in Boiotia: Regional Experiments in Technique and Iconography », Hesperia 87-2 (2018), p. 311-385 [examine une facette de la poterie béotienne décorée dans laquelle les couleurs étaient ajoutées sur le vernis noir, une pratique connue comme "the Six's technique». Discute l'iconographie - incluant notamment des scènes dionysiaques rares - en lien avec les coutumes sociales de la région].

155 SAcco L., «Il pharmakos nelle fonti antiche e nella Storia delle religioni. Alcune valutazioni critiche ", Mythos 12 (2018), p. 103-115 [s'interroge sur la signification du mot grec pharmakos et sur les origines et le déroulement de cette cérémonie rituelle].

156 SALAPATA Gina, «Tokens of Piety. Inexpensive Dedications as Functional and Symbolic Objects ", Opuscula 11 (2018), p. 97-109 [le choix d'une offrande de petite taille et/ou 
bon marché ne reflèterait pas nécessairement le statut socio-économique inférieur des dédicants].

SAMmons Benjamin, "I will send him to Crete, and sandy Pylos." A Fragment of the Cypria in the Homeric Scholia? », AClass 61 (2018), p. 201-211.

SANTAGATI E., «Rhegion arcaica e i santuari panellenici », Thiasos 8 (2019), p. 23-35 [un des aspects les plus intéressants des tyrannies archaïques de Grande Grèce est l'usage politique et religieux des ex-votos: par ces manifestations, les tyrans et citoyens de l'élite visaient à établir et/ou légitimer leur propre statut et pouvoirpolitique].

SARIPANIDI Vasiliki, «Vases, Funerary Practices, and Political Power in the Macedonian Kingdom during the Classical Period before the Rise of Philip II ", AJA 123-3 (2018), p. 381-410 [examine les témoignages funéraires - principalement la céramique allant de la période classique à l'ascension de Philippe II afin d'apporter un éclairage à cette période mal connue de l'histoire macédonienne].

savvopoulos K., « The Gold Foundation Deposit Plaque of Ptolemy IV from the Temple of Aphrodite Ourania at Koussai», ZPE 208 (2018), p. 117-120 [présentation (avec photographie) d'une plaque en or provenant du temple d'Aphrodite Ourania à Koussai]. scoтt Calloway, "Gender in the Temple: Women's Ailments in the Epidaurian Miracle Cures », ClAnt 37-2 (2018), p. 321-335 [compare les cas de maladies féminines consignés dans les récits de guérisons miraculeuses d'Épidaure (iamata) avec la théorie et la thérapeutique des textes hippocratiques afin de mesurer l'ampleur des hypothèses partagées entre le sanctuaire et la médecine hippocratique]. SEKITA Karolina, «Ades and Heracles at Pylos: Dione's Tale Dismantled », CQ 68-1 (2018), p. 1-9 [le motif du combat entre Héraclès et Hadès à Pylos, raconté par Dioné au livre $\mathrm{V}$ de l'Iliade, est particulièrement intéressant et mérite un examen minutieux]. SIEWERT Peter, « Fragment einer hocharchaischen Bronzetafel aus Olympia mit Nennung der Eleer und des Mantis-Amtes (BrU 8) », Tyche 33 (2018), p. 177-182. SHILO Amit, «The Ghost of Clytemnestra in the Eumenides: Ethical Claims beyond Human Limits ", AJPh 139-4 (2018), p. 533-576 [le fantôme de Clytemnestre dans les Euménides est la première figure de l'au-delà dans l'ensemble de la littérature grecque à demander la vengeance plutôt qu'un rituel funéraire].

165 SKEMPIS Marios, "Partheneion in Dithyramb: Bacchylides 19 ", Aevum Antiquum n.s. 16 (2016), p. 289-295. SKEMPIS Marios, « Hermes the Smooth Talker: Bacchylides 19.29 », Hermes 147-1 (2019), p.106-112 [traite d'un problème textuel dans l'ode 19 de Bacchylide. L'auteur fait l'hypothèse de la présence d'un hapax qualifiant Hermès au vers 29 (Eıpıóvioc) et propose une explication émymologique de l'épiclèse du dieu].

167 SKUSE Matthew L., " The Arcesilas Cup in Context: Greek Interactions with Late Period Funerary Art », ABSA 113 (2018), p. 221-249 [réévalue les études antérieures de la "Coupe d'Arcésilas » et ses similitudes avec les images de pesée propre à l'art funéraire égyptien]. SQUIRE D., « Supplements for Sappho frr. 27,4 and 98(a), 7 », ZPE 207 (2018), p. 15-18. STAAB Gregor, « Grabepigramm für den in Tralleis verstorbenen jungen Arzt Molon aus Hyllarima », EA 50 (2017), p. 9-16. 
170 STEWART Andrew, "Classical Sculpture from the Athenian Agora, Part 1: The Pediments and Akroteria of the Hephaisteion ", Hesperia 87-4 (2018), p.681-741 [attribue 25 sculptures fragmentaires mises au jour sur l'agora d'Athènes aux frontons et à l'akroteria de l'Hephaisteion].

171 STRAUS M., « Ritual aspects of Aristophanes' Birds »,AClass 61 (2018), p. 125-157 [traite de l'attention récente portée aux aspects rituels de l'ancienne comédie athénienne, y compris ses possibles fonctions cultuelles et civiques dans le cadre perfomatif des Grands Dionysia ; l'auteur propose l'usage de la linguistique et d'autres outils comme moyens de fournir une appréciation plus profonde de tels aspects des pièces de théâtre].

172 STRIANO Araceli, «Éros dans l'anthroponymie grecque », Mnemosyne 71-4 (2018), p. 640652 [alors qu'Éros, tout comme Aphrodite, est largement absent dans l'anthroponymie grecque aux périodes archaïque et classique, on remarque plus tard une nette augmentation, à Rome et Pompéi, mais aussi en Hispanie].

Johan,

Hesiod, Ouranos, Kronos, and the Emasculation at the Beginning of Time »,CW 111-4 (2017-2018), p. 459-484 [l'émasculation d'Ouranos par Kronos dans la Théogonie d'Hésiode serait représentée comme la genèse des saisons et des années].

URCIUOLI E.R., RÜPKE Jörg, «Urban Religion in Mediterranean Antiquity: Relocating Religious Change », Mythos 12 (2018), p. 117-135 [se concentre sur l'impact des cités sur la religion et sur la manière dont l'interaction avec l'espace urbain a changé la religion].

175 VAELSKE Veit, «Ein Widder für Ammon. Ein früher Beleg für griechische Votivpraxis in der libyschen Oase Siwa ", AA (2017-2), p. 31-47.

176 VAN SCHOOR David, "Eti zosa phlox. Inferring Divine Presence in Euripides' Bacchae », Aclass 61 (2018), p. 158-189.

177 VASSALlo Christian, «Feuer und göttliche Dialektik bei Heraklit. Neue Lesungen in P.Herc.1428, Kol. 330 (olimFr. 17) Praesocratica Herculanensia IX »,Mnemosyne 71-5 (2018), p. 717-732 [nouvelle édition critique et bref commentaire].

178 WARWICK Celsiana, "The Maternal Warrior: Gender and Kleos in the Iliad », AJPh 140-1 (2019), p. 1-28 [l'Iliade utiliserait la figure de la mère, qui représente à la fois la protection et la destruction, comme un symbole de l'instabilité des valeurs du guerrier épique, qui oscillent entre rôle protecteur et besoin de gloire (kleos)].

179 WILGAUX Jérôme, «Infirmités et prêtrise en Méditerranée antique ", Pallas 106 (2018), p. 275-287 [l'article poursuit une recherche menée précédemment sur l'intégrité attendue $\mathrm{du}$ corps des prêtres en Grèce ancienne, en s'intéressant aux normes similaires observées dans le monde juif antique].

180 ZELLMANN-ROHRER M., " Two Greek Amulets », ZPE 207 (2018), p. 105-114 [publication de deux amulettes : l'une pour laquelle une édition complète faisait encore défaut - elle a été décrite et on en a fourni un dessin et une photographie en noir et blanc, mais elle a seulement été transcrite partiellement, sans division de lignes dans Greifenhagen, Schmuckarbeiten 2 : 99) -, l'autre étant encore non publiée jusqu'à ce jour].

181 ZINGG Emmanuel, "Apollon in Knidos ", MH 75-1 (2018), p. 25-55 [la découverte à Mylasa d'une nouvelle inscription faisant mention de la migration d'Apollon Karneios de 
Sparte à Cnide offre l'occasion d'examiner les questions de la datation et des lieux de culte d'Apollon Karneios et d'Apollon Triopios à Cnide].

ZINGG Emanuel, «Ampelius 8, 16 (= I.Iasos T 34) und Erythrai », Hermes 147-1 (2019), p. 119-124 [la conjecture Argino, proposée par E. Rohde en 1877, est convaincante ; le passage fait donc référence non pas à la cité carienne de Bargylia, mais à Érythrées et à son histoire religieuse].

\section{AUTEURS}

\section{HÉLÈNE COLLARD}

Université de Liège 